Trauma Berufskrankh 2017 · 19 (Suppl 3):S231-S233 DOI 10.1007/s10039-017-0284-4

Online publiziert: 2. August 2017

๑) Springer Medizin Verlag GmbH 2017

CrossMark
E. Mayr

Klinik für Unfallchirurgie, Orthopädie, Plastische und Handchirurgie, Klinikum Augsburg, Augsburg, Deutschland

\section{Berufsgenossenschaftliches Heilverfahren und Alterstraumazentren}

\section{Braucht es neue Regelungen?}

Der derzeit stattfindende demografische Wandel führt zu einer deutlich alternden Population, die spezielle Anforderungen auch an die Traumatologie stellt. Daneben findet derzeit auch ein gesellschaftlicher Wandel statt. Waren 80-Jährige noch vor wenigen Jahrzehnten in ihrer Aktivität deutlich eingeschränkt, so sind sie heute vielfach hoch aktiv. Auch regelmäßige sportliche Betätigung ist für Hochbetagte heute durchaus ein Thema. Aber es findet derzeit auch ein sozialer Wandel in der deutschen Gesellschaft statt. Das Aufbrechen der familiären Sozialverbünde, das Folge der von der jüngeren Generation abgeforderten Mobilität ist, führt zu einer Vereinsamung unserer älter werdenden Generation. Dies bedeutet, dass im Krankheitsfall geriatrische Patienten häufig nicht mehr im sozialen Umfeld aufgefangen sind. Trotz oder vielleicht gerade wegen des medizinischen Fortschrittes häufen sich aber die chronischen Erkrankungen in unserer älter werdenden Gesellschaft. Neben mannigfaltigen Nebenerkrankungen stehen auch klassische Alterssyndrome wie Mangelernährung, Demenz, Delir, Sturzgefährdung, Osteoporose und chronische Schmerzen im Fokus der Patienten. Sie müssen in der Behandlung der geriatrischen Traumapatienten Berücksichtigung finden.

All diese genannten Entwicklungen haben in den vergangenen Jahren dazu geführt, dass vielerorts spezialisierte Zentren für die Behandlung von geriatrischen Traumapatienten gegründet worden sind. Kern dieser Alterstraumazentren ist dabei eine interdisziplinäre und interprofessionelle Behandlung der entsprechenden Patienten mit entsprechenden innovativen Ansätzen. Abweichend von der allgemeinen Traumatologie haben sich für die Alterstraumatologie 4 Säulen herauskristallisiert, an denen sich die traumatologische Behandlung orientiert. Dieses sind

1. Vollbelastbarkeit,

2. Single-shot-Surgery,

3. Mobilität geht vor Funktionalität,

4. eine an den individuellen Bedürfnissen des Patienten orientierte Medizin.

Diese „Credos der Alterstraumatologie“ führen aktuell und werden auch in den kommenden Jahren zu neuen operativen Verfahren führen.

Natürlich sind alterstraumatologische Patienten nicht der Fokus einer bg-lich (berufsgenossenschaftlich) versicherten Patientenklientel. Neben den klassischen älteren Patienten, die bg-lichen Versicherungsschutz genießen, insbesondere aus dem landwirtschaftlichen Arbeitsumfeld, wird sich hier in den kommenden Jahren jedoch eine Änderung ergeben, ist doch eine Vielzahl von Rentnern heute auf einen Mini-Job, der dann auch berufsgenossenschaftlichen Versicherungsschutz genießt, angewiesen. Im eigenen Krankengut machen die über 70Jährigen etwa $2 \%$ aller bg-lich stationären Patienten aus.

\section{Nutzen interdisziplinärer Zentren für Alterstraumatologie}

Der Nutzen solcher interdisziplinärer Zentren für Alterstraumatologie wird derzeit im Rahmen mehrerer Studien untersucht. Im Jahr 2010 hat Bachmann [1] eine Metaanalyse von randomisierten Kontrollstudien vorgelegt, die eindeutig zeigt, dass die Behandlung geriatrisch traumatologischer Patienten in entsprechenden spezialisierten Zentren zu einer signifikanten funktionellen Verbesserung des Outcomes führt. Auch die Rückführung entsprechender Traumapatienten in die häusliche Umgebung ist nach einer Behandlung in einem Alterstraumazentrum signifikant wahrscheinlicher zu erreichen als bei einer Behandlung in einer regulären Traumaeinheit. Im Übrigen zeigte sich auch eine signifikante Reduktion der Mortalität bis $\mathrm{zu} 12$ Monaten nach dem Unfall.

\section{Kosten}

Nachdem also eine hohe Relevanz vonseiten der Patienten für eine Behandlung in einem spezialisierten Alterstraumazentrum besteht, stellt sich die Frage nach dem Kostenpunkt. Im eigenen Krankenhaus haben wir 2010 eine 44Betten-Einheit für interdisziplinäre und interprofessionelle Alterstraumatologie gegründet und in Betrieb genommen. Entsprechend den Vorgaben des Bayerischen Geriatriekonzeptes sowie den Vorgaben, die sich aus dem DRG-Sys- 
tem ableiten lassen, mussten neben baulichen Maßnahmen insbesondere personelle Adjustierungen vorgenommen werden. Insgesamt summieren sich dabei die jährlichen Personalmehrkosten für den Betrieb dieser 44-BettenAlterstraumatologie auf ca. $550.000 €$. Zur Gegenfinanzierung dieser Personalmehrkosten ist im DRG-System die sog. geriatrische frührehabilitative Komplexbehandlung vorgesehen. Auch wenn es für diese Komplexbehandlung 3 unterschiedliche, von der Behandlungsdauer und der Anzahl der Therapieeinheiten abhängige Unterstufungen gibt, so ist lediglich eine stationäre Behandlung unter GFK(geriatrische frührehabilitative Komplexbehandlung)-Kriterien ab einer Mindestdauer von 14 Tagen und mindestens 20 erbrachten Therapieeinheiten erlössteigernd. Werden diese beiden Kriterien (Liegedauer und Anzahl der Therapieeinheiten) nicht erfüllt, die Behandlung aber dennoch zum Wohle des Patienten unter den übrigen GFKBedingungen (interdisziplinäres Team, aktivierend therapeutische Pflege, hoher therapeutischer Anteil der Behandlung, geriatrisches Assessment, regelmäßige Therapiebesprechungen im Team ...) erbracht, so wird dieser für den Leistungserbringer erhebliche Mehraufwand nicht refinanziert.

\section{Forderungen für die Zukunft}

Abgeleitet von Absatz 2 des $\$ 1$ des SGB VII, in dem festgehalten ist, dass es die Aufgabe der gesetzlichen Unfallversicherung ist, nach Eintritt von Arbeitsunfällen und Berufskrankheiten die Gesundheit und die Leistungsfähigkeit der Versicherten mit allen geeigneten Mitteln wiederherzustellen, muss die Forderung erhoben werden, dass die gesetzlichen Unfallversicherungsträger sich künftig eindeutig dazu positionieren, dass ihre Versicherten künftig in entsprechenden Zentren für Alterstraumatologie behandelt werden, ist doch der Nutzen dieser spezialisierten Einheiten in der Zwischenzeit wissenschaftlich nachgewiesen. Die Beteiligung der Krankenhäuser an einem solchen Verfahren sollte dabei unabhängig von der bisherigen Abstufung DAV (Durchgangsarztverfahren)

Trauma Berufskrankh 2017 · 19 (Suppl 3):S231-S233 DOI 10.1007/s10039-017-0284-4

(c) Springer Medizin Verlag GmbH 2017

\section{E. Mayr \\ Berufsgenossenschaftliches Heilverfahren und Alterstraumazentren. Braucht es neue Regelungen?}

\section{Zusammenfassung}

Die demografische Entwicklung unserer Gesellschaft bringt Veränderungen mit sich, auf die sich die Traumatologie einstellen muss. Vielerorts sind in den vergangenen Jahren spezialisierte Alterstraumazentren entstanden, in denen in interdisziplinärer und interprofessionaler Zusammenarbeit die Behandlung geriatrischer Traumapatienten erfolgt. Wissenschaftlich konnte der Nutzen solcher Zentren gegenüber der Standardbehandlung in der Zwischenzeit nachgewiesen werden. Abgeleitet vom $\S 1$ des SGB VII müssen die gesetzlichen Unfallversicherungsträger damit darauf drängen, dass ihre Versicherten in eben solchen Einrichtungen behandelt werden, die über ein entsprechendes Zertifikat verfügen. Das Zertifikat selbst sollte von den beiden Fachgesellschaften für Unfallchirurgie und Geriatrie gemeinsam erarbeitet werden, da die Zusammenarbeit der beiden Fächer eben solche Alterstraumazentren ausmacht. Die Möglichkeit zur Beteiligung an einem stationären Heilverfahren für geriatrische Traumapatienten sollte dabei unabhängig von der dreistufigen Einordnung DAV (Durchgangsarztverfahren) - VAV (Verletzungsartenverfahren) - SAV (Schwerstverletzungsartenverfahren) möglich sein. Allerdings muss auch eine adäquate Finanzierung für diese kostenintensive Behandlung sichergestellt werden.

Schlüsselwörter

Traumatologie - Geriatrie - Zertifikat . Finanzierung $\cdot$ Behandlung

\section{Occupational insurance association treatment and trauma centers for the elderly. Are new regulations necessary?}

\section{Abstract}

The demographic developments in our society implicates alterations which traumatology must adjust to. In many places specialized trauma centers for the elderly have been established in recent years, in which an interdisciplinary and interprofessional cooperation is carried out for the treatment of geriatric trauma patients. Scientifically, the benefits of such centers could in the meantime be proven compared to standard treatment. As derived from $\S 1$ of the Social Code Book VII, the statutory accident insurances must therefore insist that their insurees are treated in such an establishment, which are in possession of an appropriate certificate. The certificate should be should be jointly processed by the specialist societies for trauma surgery and geriatrics, because the cooperation of these two disciplines forms the basis for such trauma centers for the elderly. The possibility for participation in an inpatient treatment procedure for geriatric trauma patents should be independent of the 3-step classification of accident insurance consultant (DAV), injury type procedure (VAV) and most severe injury type procedure (SAV); however, an adequate funding for this cost-intensive treatment must also be ensured.

\section{Keywords}

Traumatology · Geriatrics · Certificate . Funding · Treatment
- VAV (Verletzungsartenverfahren) SAV (Schwerstverletzungsartenverfahren) sein, sondern vielmehr von einem entsprechenden Zertifikat. Ob das derzeit existierende Zertifikat der Deutschen Gesellschaft für Unfallchirurgie (DGU) hierfür ausreicht oder ob hier nicht ein gemeinsames Zertifikat von DGU und Deutscher Gesellschaft für Geriatrie zusammen entwickelt werden sollte, muss dabei derzeit noch offen bleiben. Einiges spräche jedoch dafür, auf ein gemeinsames, von Unfallchirurgen und Geriatern entwickeltes Zertifikat zu bestehen, ist doch eben die Zusammenarbeit dieser beiden Berufsgruppen auf Arbeitsebene der Garant für den gewünschten Erfolg. Um einen entsprechenden Anreiz zu schaffen, sollte aber künftig auch die sog. Kurz-GFK erlössteigernd sein, da aktive Patienten in einem entsprechenden Alter auf der einen Seite nicht zwingend län- 
ger als notwendig hospitalisiert werden sollten, auf der anderen Seite aber von der intensiven, interdisziplinären und interprofessionellen Betreuung in einem Alterstraumazentrum profitieren.

\section{Fazit für die Praxis}

- Zukünftig sollte die stationäre Behandlung geriatrischer Traumapatienten im Rahmen der gesetzlichen Unfallversicherung nur noch in einem zertifizierten Traumazentrum stattfinden.

- Die Beteiligung der stationären Einrichtungen an diesem Verfahren sollte nur von einem entsprechenden Zertifikat und nicht von der Versorgungsstufe der stationären Einrichtung abhängen.

\section{Korrespondenzadresse}

\section{Prof. Dr. Dr. h.c. E. Mayr}

Klinik für Unfallchirurgie, Orthopädie,

Plastische und Handchirurgie, Klinikum

Augsburg

Stenglinstr. 2, 86156 Augsburg, Deutschland

Edgar.Mayr@klinikum-augsburg.de

\section{Einhaltung ethischer Richtlinien}

Interessenkonflikt. E. Mayr gibt an, dass kein Interessenkonflikt besteht.

Dieser Beitrag beinhaltet keine vom Autor durchgeführten Studien an Menschen oder Tieren.

The supplement containing this article is not sponsored by industry.

\section{Literatur}

1. Bachmann $S$, Finger $C$, Huss A, Egger M, Stuck AE, Clough-Gorr KM (2010) Inpatient rehabilitation specifically designed for geriatric patients:systematic review and meta-analysis of randomized controlled trials. BMJ 340:c1718. doi:10.1136/bmj.c1718 